\title{
Prediction is Difficult, Especially About the Future: Clinical Prognostic Tools in Melanoma
}

\author{
Vernon K. Sondak, $\mathrm{MD}^{1,2}$ and Jane L. Messina, $\mathrm{MD}^{1,2,3,4}$ \\ ${ }^{1}$ Department of Cutaneous Oncology, Moffitt Cancer Center, Tampa, FL; ${ }^{2}$ Department of Oncologic Sciences, University \\ of South Florida Morsani College of Medicine, Tampa, FL; ${ }^{3}$ Department of Anatomic Pathology, Moffitt Cancer Center, \\ Tampa, FL; ${ }^{4}$ Departments of Pathology and Cell Biology, Dermatology and Oncologic Sciences, University of South \\ Florida Morsani College of Medicine, Tampa, FL
}

"What are my chances, doc?" No surgical oncologist who has been in practice more than a few months has escaped being asked this question by an anxious patient. Yet it is rare that the answer addresses the anxiety of the questioner, and rarer still that the answer accurately reflects the future course of the patient's malignancy, especially when the patient has been diagnosed with cutaneous melanoma. Despite the progress recent years have seen in the understanding of melanoma biology and the consequent advances in therapy, our ability to assess the likelihood of recurrence and death for any individual patient remains conspicuously limited. Conventional staging systems such as the ubiquitous American Joint Committee on Cancer (AJCC) classification can subdivide patients with clinically localized (stage I through III) melanoma into those at relatively low, average, or high risk of recurrence and death, but, even then, at least $10-20 \%$ of patients predicted to be at low or high risk of recurrence will defy the odds and their tumors will behave contrary to expectations. Moreover, as the incidence of melanoma continues to increase and more and more patients are diagnosed with early-stage disease, the absolute number of patients presenting with 'low risk' melanoma who ultimately relapse and die is growing. ${ }^{1,2}$ Sentinel lymph node biopsy (SLNB) clearly represented a major step forward in prognostication for patients with clinically localized cutaneous melanoma, ${ }^{3}$ but if we are ever to dramatically decrease the death toll from

\footnotetext{
(C) Society of Surgical Oncology 2016
}

First Received: 26 February 2016;

Published Online: 19 May 2016

V. K. Sondak, MD

e-mail: vernon.sondak@moffitt.org melanoma via adjuvant therapy strategies, then we must find ways to identify the 'bad actors' among the patients who the AJCC staging system would classify as at low to moderate risk for recurrence.

For patients with localized melanoma, the AJCC staging system uses only a few clinicopathologic factors (tumor thickness, presence or absence of ulceration and/or mitoses [for T1 lesions only], regional lymph node status, number of nodal metastases, and how those nodal metastases were identified) to assign a stage and confer an expected incidence of recurrence and death from melanoma. Many prognostic models have been constructed that utilize additional, readily available features, such as patient age and sex, anatomic site of the primary, histologic subtype of the primary, presence and pattern of tumor-infiltrating lymphocytes and status of the nonsentinel nodes, to name only a few, to allow the calculation of a more refined individual prediction. These models may be used to create a nomogram, wherein a numerical value from the sum of various individual prognostic factors is used to graphically derive an individual's risk of recurrence or death. More recently, electronic calculators, often using web-based data entry, offer patients or providers an immediate assessment of the likelihood of an adverse or successful outcome. These tools clearly address the patient's question posed at the start of this editorial, but do they do so accurately?

Mahar and colleagues set out to answer this important question, ${ }^{4}$ and did so in a commendably rigorous and thorough fashion. They evaluated 17 different clinical prognostic tools for stage I-III primary cutaneous melanoma, $75 \%$ of which used data from patients diagnosed at least 10 years ago (2006 or earlier), and fewer than half of which had undergone any form of external validation. Not 
surprisingly, they found little consistency in the data elements used to construct the various prognostic models: 14 different prognostic factors were included in at least two of the 17 tools, and 12 prognostic factors were included in only one tool. ${ }^{4}$ For only 8 of the 17 tools, model performance was externally validated in an independent sample of data, and in just 3 of them, at least two different datasets were used for the external validation. ${ }^{4}$ The findings of this analysis convincingly support the authors' conclusions that there is widespread need for methodological improvements in the development and evaluation of the external generalizability and clinical impact of prognostic tools in melanoma.

Equally important as what the authors studied is what they could not study in a similarly rigorous fashion; this comprehensive analysis of prognostic models and tools did not address emerging prognostic factors such as immunohistochemical determination of proliferative index, mutational analysis such as BRAF status, evaluation of the immune microenvironment, or DNA, RNA, gene or protein expression profiles/signatures. The potential for these new technologies to revolutionize melanoma prognostication is intuitively obvious, and we have already seen some of these technologies become commonplace in other tumor types, particularly breast cancer. ${ }^{5}$ Closer to home, molecular prognostication has become commonplace for patients with uveal melanoma. Defining prognostic groups in uveal melanoma poses different challenges than we face for cutaneous melanoma, including the lack of comprehensive histopathologic analysis of the primary in cases where the tumor is treated by radiation rather than enucleation, and the absence of regional lymphatic metastasis for most eye tumors. In an attempt to fill this void, molecular prognosticators were tested and quickly found to have a credible scientific basis in the biology of the disease. ${ }^{6,7}$ Unlike any of the cutaneous melanoma clinical prognostic tools Mahar et al. analyzed, ${ }^{4}$ a 15 -gene expression profile that could classify risk for patients with uveal melanoma was prospectively validated in a multicenter study involving 459 patients from 12 independent centers. ${ }^{8}$ This gene expression profile has now been commercialized and is offered to many patients with uveal melanoma (DecisionDx-UM; Castle Biosciences, Phoenix, AZ, USA). In fact, its success has added a new twist to the push for prognostication; we know the assay adds prognostic information but we do not know what to do differently for patients based on this information. Moreover, real-world experience with this prognostic test has shown that patients frequently find the knowledge that they are in a high-risk group with very little that can be done about it more unsettling than helpful, at least without detailed and sometimes extensive counseling.
A similar (but genetically unrelated) gene expression profile test is now commercially available for cutaneous melanoma (DecisionDx-Melanoma; Castle Biosciences). This test has not yet been evaluated in the kind of prospective clinical study that demonstrated the value of the uveal melanoma profile, nor is the scientific basis for including the component genes as well characterized, but evaluations of archival tissue collections have demonstrated independent statistical significance as a predictor of metastatic risk compared with AJCC stage or tumor thickness. ${ }^{9}$ The clinical value of this test remains unclear, and the National Comprehensive Cancer Network (NCCN) Guidelines for Melanoma state "while there is interest in newer prognostic molecular techniques such as gene expression profiling to differentiate... melanomas at lowversus high-risk for metastasis, routine (baseline) genetic testing of primary cutaneous melanomas (before or following SLNB) is not recommended outside of a clinical study (trial)." ${ }^{10}$ This admonition seems sound, particularly if we apply the same stringent criteria for validation that Mahar et al. applied to clinical prognostic tools that also claim to demonstrate independent prognostic value. Nevertheless, practicing surgical oncologists are encountering patients with clinically localized invasive melanomas who have already undergone this test at the time of referral. For the time being, until far more detailed data derived from prospective evaluations are available, there is insufficient evidence to propose that routine clinical management, including decision making regarding SLNB, should be altered by the results of gene expression profiling, although to reassure patients who have already received and are concerned about a "high-risk" determination, more intensive surveillance may be justified. In fact, the lessons learned from this survey of prognostic tools can and should be taken into account when considering how to integrate new prognostic tools such as this molecular assay into clinical practice.

Along with the explosion of molecular techniques that may ultimately be able to predict melanoma prognosis, advances in bioinformatics, data mining, and shared tissue resources such as The Cancer Genome Atlas (TCGA) are increasing our capability to understand the fundamental genetic and epigenetic determinants of a tumor's ability to metastasize and kill. Some of the shortcomings of currently available prognostic tools highlighted by Mahar et al., such as relatively small study populations, inclusion of limited numbers of variables, short follow-up, and lack of internal and external validation, can be overcome with careful planning and buy-in from academic thought leaders and oncology research cooperative groups. Taking into account lessons being learned from the currently commercially available molecular assays, such studies should evaluate the cost effectiveness of any alterations in follow-up 
interval or the addition of imaging studies in higher risk patients. The creation of large, prospective, multi-institution registry studies that can harness the power of electronic data sharing should be a goal of our field as we move forward into the era of personalized medicine. Nevertheless, we should always keep in mind the ultimate goal of these efforts-to finally be able to answer our melanoma patients' most pressing question.

DISCLOSURES Dr. Sondak is a paid consultant for BMS, Genentech/Roche, Merck, and Novartis. Dr. Messina declares no financial interests.

\section{REFERENCES}

1. Gimotty PA, Guerry D. Prognostication in thin cutaneous melanomas. Arch Pathol Lab Med. 2010;134:1758-63.

2. Whiteman DC, Baade PD, Olsen CM. More people die from thin melanoma $(\leq 1 \mathrm{~mm})$ than from thick melanoma $(>4 \mathrm{~mm})$ in Queensland, Australia. J Invest Dermatol. 2015;135:1190-3.
3. Morton DL, Thompson JF, Cochran AJ, et al. Final trial report of sentinel-node biopsy versus nodal observation in melanoma. $N$ Engl J Med. 2014;370:599-609.

4. Mahar AL, Compton C, Halabi S, et al. Critical assessment of clinical prognostic tools in melanoma. Ann Surg Oncol. 2016. doi:10.1245/s10434-016-5212-5.

5. Győrffy B, Hatzis C, Sanft T, Hofstatter E, Aktas B, Pusztai L. Multigene prognostic tests in breast cancer: past, present, future. Breast Cancer Res. 2015;17:11.

6. Onken MD, Worley LA, Tuscan MD, Harbour JW. An accurate, clinically feasible multi-gene expression assay for predicting metastasis in uveal melanoma. J Mol Diagn. 2010;12:461-8.

7. Harbour JW, Onken MD, Roberson EDO, et al. Frequent mutation of BAP1 in metastasizing uveal melanoma. Science. 2010;330:1410-3.

8. Onken MD, Worley LA, Char DH, et al. Collaborative Ocular Oncology Group report no. 1: prospective validation of a multigene prognostic assay in uveal melanoma. Ophthalmology. 2012;119:1596-603.

9. Gerami P, Cook RW, Wilkinson J, et al. Development of a prognostic genetic signature to predict the metastatic risk associated with cutaneous melanoma. Clin Cancer Res. 2015;21;175-83.

10. NCCN Clinical Practice Guidelines in Oncology: Melanoma (version 2.2016). Fort Washington (PA): National Comprehensive Cancer Network; 2016. 